\title{
Performance Evaluation of Data Mining based Images by using Fuzzy, Mean, Median Trilateral Filter
}

\author{
Kuljeet Kaur \\ M.Tech Student \\ Amritsar College of Engineering and Technology \\ Amritsar
}

\author{
Aarti \\ Assistant Professor \\ Amritsar College of Engineering and Technology \\ Amritsar
}

\begin{abstract}
In Digital Image Processing removing the noise from an images is a very important to get the excellent result. Different filtering techniques like Median Filter and Mean Filter is not effective oftentimes for filtering the digital images. The newest procedure in this paper has focused on the data mining methods to improve data mining based fuzzy filtering further by utilizing filter for mixed noises and adaptive manifolds and high-dimensional mean-median filter for salt and pepper noises for successfully removing the noise. The latest working in this paper is that the usage of Trilateral filter for filtering the images, it is especially uses when a Gaussian noise is created in the images. The performance is evaluated by applying, Peak Signal to noise ratio, Root mean square error, Normalized cross-co relation it shows encouraging results.
\end{abstract}

\section{Keywords}

Digital Image Processing, Multiplicative Noisy Images, Trilateral Filter, Root Mean square error, Peak signal to noise ratio, Normalized cross-co relation, Data Mining.

\section{INTRODUCTION}

Digital image processing could be the usage of using formulations to execute photograph managing on digital images. As a subcategory in addition to issue linked to digital managing, digital image processing has a few benefits about analog influence processing. It permits a considerably higher choice of formulation to acquire applied towards understanding data and reduce problems. Data mining can be an interdisciplinary subfield of pc science. It could be the computational procedure for exploring styles in big information models concerning techniques at the junction of synthetic intelligence, equipment understanding, statics and repository systems.

The overall purpose of the data mining method is always to acquire data from the information collection and change it in to a clear design for more use. The connected phrases knowledge, data mining and knowledge snooping reference the utilization of information mining solutions to trial areas of a larger information collection which can be may possibly also little for trusted statistical inferences to be manufactured in regards to the validity of any designs discovered. Data Mining requires five frequent courses of tasks.

- Anomaly Recognition

- Association

- $\quad$ Clustering

- Classification

- Regression

\section{NOISE}

\subsection{Gaussian Noise}

Removing with larger common deviations suppresses sound, but additionally blurs the image. Gaussian noise is mathematical sound hiring a likelihood occurrence purpose (PDF) similar compared to that in the conventional writing, that's also called the Gaussian is submitting. In few different words, the values the way the noise usually considers are generally Gaussian-distributed.

\subsection{Salt and pepper Noise}

The Salt \& Pepper noise is observed on images. It occurs as occurring bright and black pixels.

\subsection{Multiplicative Noise (Speckle Noise)}

Speckle is a granular' noise 'that inherently exists in and degrades the rank of the powerful radar, medical ultrasound and visible coherence tomography images. The truly amazing most of products, synthetic or usual, are very difficult on the product range of the wavelength.

\section{FILTER}

A fresh algorithm is presented that may remove behavioral instinct noise by corrupted pictures while conserving details. The algorithm will depend on fuzzy behavioral instinct detection along with fuzzy disturbance cancellation techniques.

\subsection{Trilateral Filter}

Trilateral filter offers tougher noise decrease and greater outlier rejection in large gradient parts and it decrease the edge-limited removing behavior of shock-forming PDE's by area obtaining with an easy min-max stacks. Trilateral filtration quickly also includes N-Dimensional signs ,however additionally it presents greater efficiency for most visible purposes including appearance-preserving distinction decrease issues for electronic images and denoising polygonal works

\subsection{Mean Filter}

The mean filtration is really an easy sliding-window spatial filtration that changes the guts price in the screen with the common (mean) of all of the pixel prices in the window.

\subsection{Median Filter}

The median filtration can be a sliding-window spatial filtration; nonetheless it changes the price in the screen with the median of all pixel prices in the window. When it comes to mean filtration, the kernel is normally square but could be any shape.

\section{PROPOSED METHOD}

This work mainly focuses on the denoising and edgepreservation of different images. Different denoising methods 
have been proposed but there is some scope of improvement. Noise is any unwanted signal that often gets added to the images when an image is being acquired or transmitted. In this planned work there is a use of Trilateral Filter is to be used for removing and detecting the noises from images also use for edge-preservation.

The proposed method involves the working as follows:

Step 1: First of all take an input image.

Step 2: Evaluate the noise type using Fuzzy rules.
Step 3: If there is a Gaussian Noise type is create then apply the Trilateral Filter. If there is a salt and pepper noise is create then apply Adaptive Manifolds and High-Dimensional MeanMedian Filter. If there is a mixed noise is created then applies Fuzzy Filter.

Step 4: After apply the filters, noise free image is created.

Step 5: Apply border correction for edge preservation.

Step 6: All steps to be completed Final image is output.

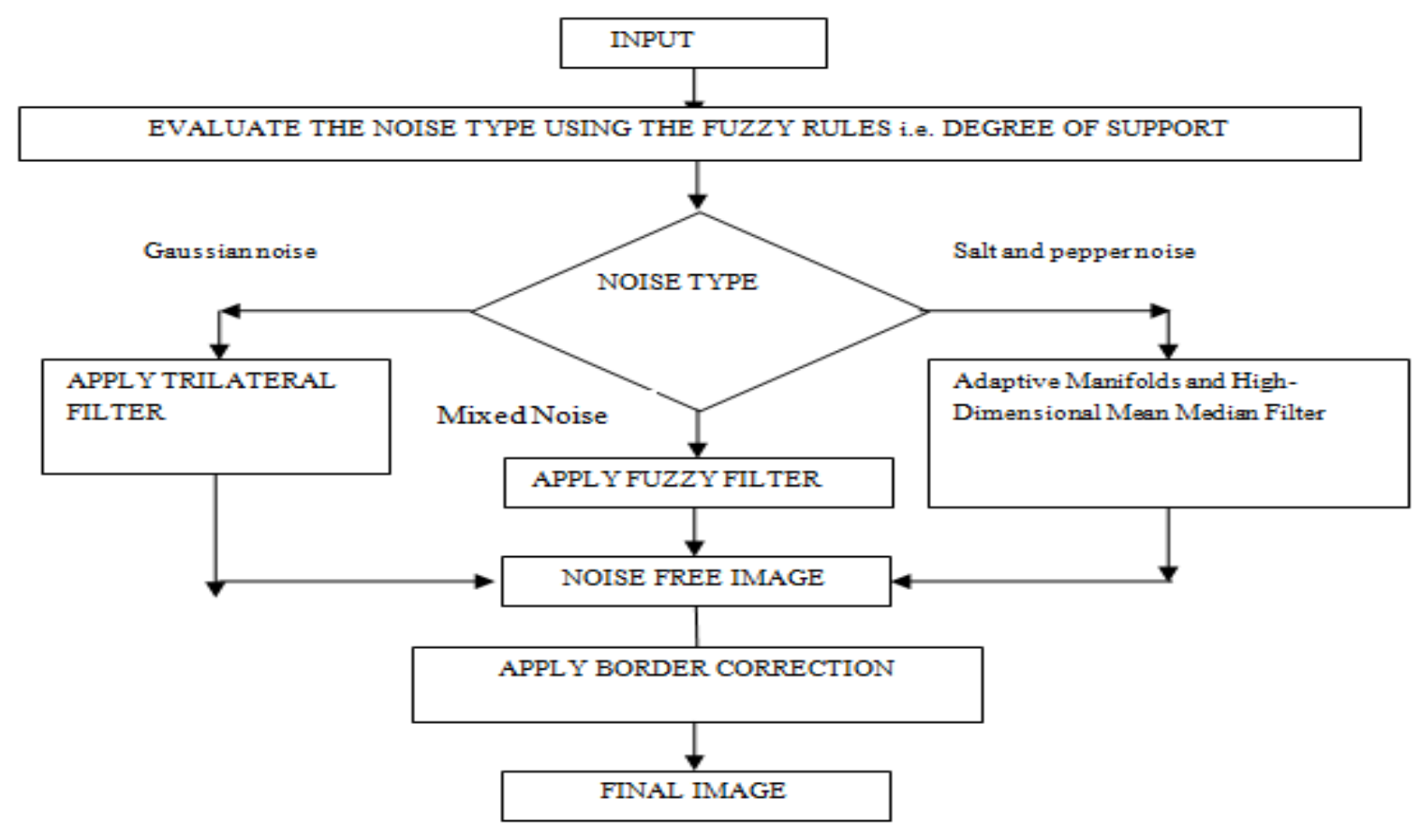

Fig 1: Flow chart of proposed algorithm

\section{RESULTS AND DISCUSSION}

For the fresh benefits planned algorithm, style and implementation has been conducted in MATLAB applying picture handling toolbox.

The figure 2 below shows three original input images of without the addition of noise. The noise create in the image usually disturbs the quality of an image.

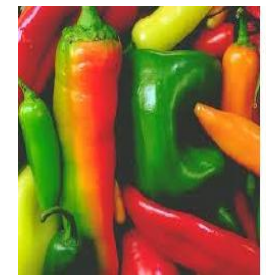

a) Pepper Image

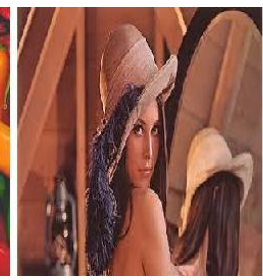

b) Lena Image

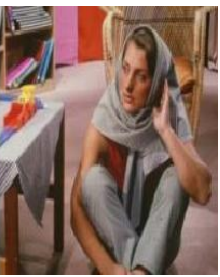

c) Barbara Image
Fig 2: Original Images

The figure 3 below shows the images in which are addition of noise. These three images is corrupted by $50 \%$ noise density.
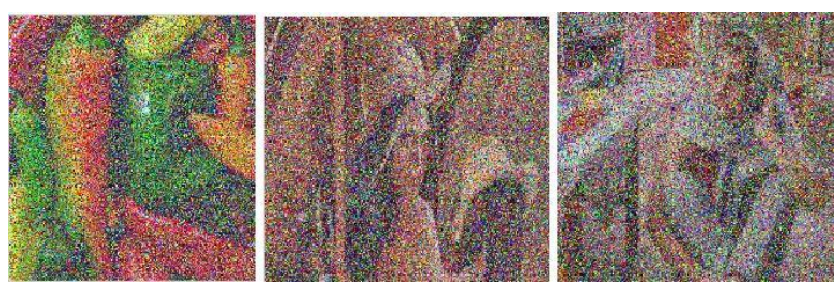

a) Pepper Image

b) Lena Image

c) Barbara Image

Fig 3: Noisy Images with $50 \%$ noise corrupted

The figure 4 below shows the output results of the existing method which is afflicted with noise. These images are achieved by applying Mean-Median Filter on the input image which is noisy.

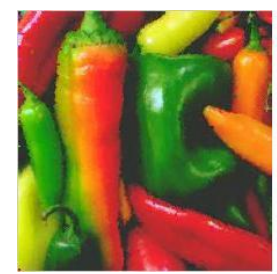

a) Pepper Image

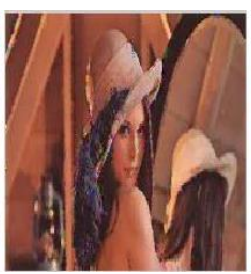

b) Lena Image

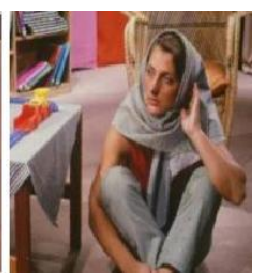

c) Barbara Image
Fig 4: Existing Result Images

The figure 5 shown below is the final stage of our proposed work in which the edge preservation and denoising results are 
shown. In the last step of our proposed work, trilateral filter is applied after applying the Mean-Median filter. The result shows that the proposed work gives best results than the existing methods
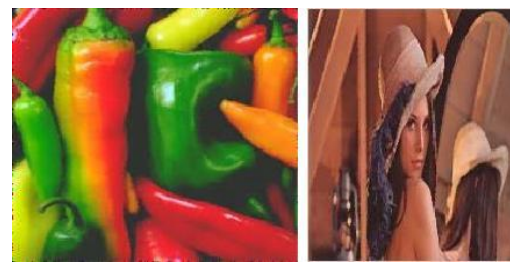

a) Pepper Image b) Lena Image

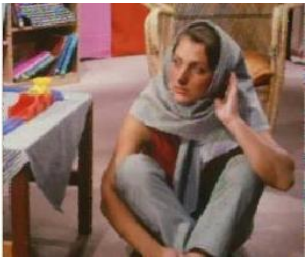

c) Barbara Image
Fig 5: Proposed Output Images

\section{PERFORMANCE EVALUATION}

In that portion, an examination has been offered between the prevailing approach and the planned work with the foundation of contrast involving the three identified parameters. The relative examination can make certain that the planned approach increases results than the prevailing one.

\subsection{Peak Signal to Noise Ratio}

Peak square noise ratio is the ratio between the maximum possible value of the signal and the power of the corrupting noise. It is measured in decibels (db). It can be explained as:

$$
\text { 10. } \log _{10}\left(\frac{M A X I^{2}}{M S E}\right)
$$

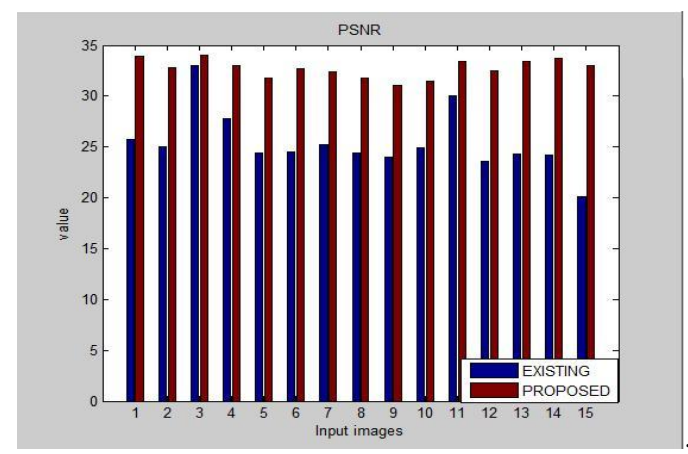

Fig 6: Graph for PSNR

In the above figure shows a plot between the existing technique shown in blue colour and the proposed technique shown in red colour. It clearly states that the proposed work shows good results as it shows increasing values for various images for PSNR.

The table 1 given below shows the values for both existing as well as proposed method. The values for PSNR should be increasing for each image for the proposed method, so it is clear from the comparison table that values for existing method are less and for proposed method are more.

Table1. Peak Signal to Noise Ratio

\begin{tabular}{|c|c|c|}
\hline Image No. & $\begin{array}{c}\text { Existing } \\
\text { Results }\end{array}$ & $\begin{array}{c}\text { Proposed } \\
\text { Results }\end{array}$ \\
\hline Image 1 & 25.7628 & 33.8962 \\
\hline Image 2 & 25.0314 & 32.8204 \\
\hline Image 3 & 32.9778 & 34.0044 \\
\hline Image 4 & 27.8267 & 33.0003 \\
\hline
\end{tabular}

\begin{tabular}{|c|c|c|}
\hline Image 5 & 24.4565 & 31.7419 \\
\hline Image 6 & 24.5328 & 32.6597 \\
\hline Image 7 & 25.2252 & 32.3509 \\
\hline Image 8 & 24.4383 & 31.7305 \\
\hline Image 9 & 23.9807 & 31.0339 \\
\hline Image 10 & 24.9254 & 31.5143 \\
\hline Image 11 & 30.0258 & 33.3816 \\
\hline Image 12 & 23.5914 & 32.5313 \\
\hline Image 13 & 24.2903 & 33.3650 \\
\hline Image 14 & 24.1789 & 33.7008 \\
\hline Image 15 & 20.1142 & 32.9808 \\
\hline
\end{tabular}

\subsection{Normalized cross-co relation}

These measures measure the similarity between the two different images; hence, they are complementary to the difference based measures. As difference measure and correlation measures complement each other.

$$
N C C=\frac{\sum \sum[I(U, V) * 0(U, V)]}{\sum \sum[I(U, V) * I(U, V)]}
$$

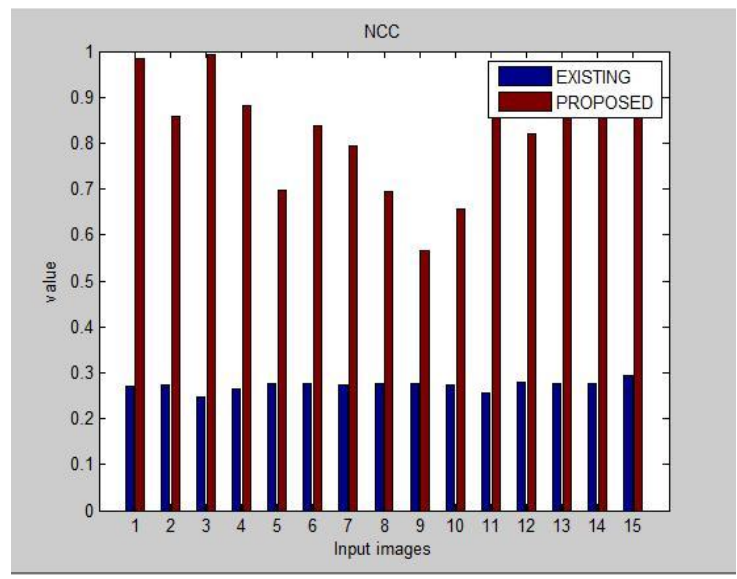

Fig 8: Graph for NCC

The above shown figure shows a comparison in the form of graph in which existing values are shown in blue colour and the values for proposed method are shown in red colour. As it is clear from the plot that proposed method shows improved results.

The table 2 given below shows the values for both existing as well as proposed method. The values for Normalized cross-co relation should be increasing for each image for the proposed method, so it is clear from the comparison table that values for existing method are less and for proposed method are more.

Table 2. Normalized cross-co relation

\begin{tabular}{|c|c|c|}
\hline Image No. & $\begin{array}{c}\text { Existing } \\
\text { Results }\end{array}$ & $\begin{array}{c}\text { Proposed } \\
\text { results }\end{array}$ \\
\hline Image 1 & 0.2707 & 0.9828 \\
\hline Image 2 & 0.2734 & 0.8578 \\
\hline Image 3 & 0.2466 & 0.9939 \\
\hline
\end{tabular}




\begin{tabular}{|c|c|c|}
\hline Image 4 & 0.2633 & 0.8808 \\
\hline Image 5 & 0.2755 & 0.6973 \\
\hline Image 6 & 0.2752 & 0.8364 \\
\hline Image 7 & 0.2726 & 0.7931 \\
\hline Image 8 & 0.2756 & 0.6954 \\
\hline Image 9 & 0.2773 & 0.5650 \\
\hline Image 10 & 0.2738 & 0.6575 \\
\hline Image 11 & 0.2559 & 0.9266 \\
\hline Image 12 & 0.2789 & 0.8188 \\
\hline Image 13 & 0.2762 & 0.9247 \\
\hline Image 14 & 0.2766 & 0.9622 \\
\hline Image 15 & 0.2931 & 0.8784 \\
\hline
\end{tabular}

\subsection{Root Mean Square Error}

Root-mean-square error is a measure of the differences between values predicted by a model or an estimator and the values actually observed. It can be explained as:

$$
\text { RMSE }=\sqrt{\frac{1}{\mathrm{KL}} \sum_{\mathrm{U}=1}^{\mathrm{K}} \sum_{\mathrm{V}=1}^{\mathrm{L}}\left(\mathrm{G}(\mathrm{U}, \mathrm{V})-\mathrm{G}^{\prime}(\mathrm{U}, \mathrm{V})\right)^{2}}
$$

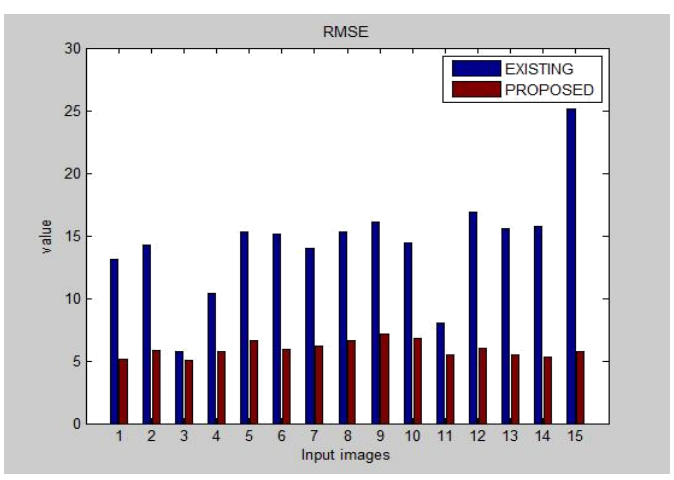

Fig 8: Graph for RMSE

Figure shows the analysis of RMSE of different images by showing the values of existing method shown in blue colour and the proposed method shown in red colour. The above graph states clearly that the proposed work shows better results than the existing work.

The values of Root Mean Square Error are given below in the comparison table 3.Table indicates the quantized examination of the Root Mean Square Error of various photographs by Existing value \& planned values. It's specific from the plan that there surely is reduction in RMSE value of photos with the usage of planned strategy around different methods. That reduces shows development in the target quality of the image. This RMSE chart shows that the prices of planned algorithm is minimal than the prevailing algorithm which.

Table 3. Root Mean Square Error

\begin{tabular}{|c|c|c|}
\hline Image No. & $\begin{array}{c}\text { Existing } \\
\text { Results }\end{array}$ & $\begin{array}{c}\text { Proposed } \\
\text { Results }\end{array}$ \\
\hline Image 1 & 13.1341 & 5.1491 \\
\hline
\end{tabular}

\begin{tabular}{|c|c|c|}
\hline Image 2 & 14.2880 & 5.8280 \\
\hline Image 3 & 5.7233 & 5.0854 \\
\hline Image 4 & 10.3564 & 5.7085 \\
\hline Image 5 & 15.2657 & 6.5985 \\
\hline Image 6 & 15.1321 & 5.9369 \\
\hline Image 7 & 13.9727 & 6.1517 \\
\hline Image 8 & 15.2977 & 6.6072 \\
\hline Image 9 & 16.1252 & 7.1589 \\
\hline Image 10 & 14.4634 & 6.7737 \\
\hline Image 11 & 8.0399 & 5.4634 \\
\hline Image 12 & 16.8643 & 6.0252 \\
\hline Image 13 & 15.5605 & 5.4738 \\
\hline Image 14 & 15.7613 & 5.2662 \\
\hline Image 15 & 25.1669 & 5.7213 \\
\hline
\end{tabular}

\section{CONCLUSION}

In this research work filtration methods based on image denoising techniques are given. Conventional filtering techniques like median filter and mean filter have not given the better results. This research work has focused on data mining based filtering techniques to check noise type and proposes a more rewarding trilateral filter it also improves median filter and mean filter. Different types of images such as grey scale or colored images are taken for experimental results. Trilateral filter, improved median filter, Mean filter gives better results as compare to the previous technique. These filters remove the noise at high noise density and preserve the edge of the grey scale as well as colored images.

\section{ACKNOWLEDGMENTS}

I am highly grateful to "Er. Aarti" for providing this opportunity. It was purely on the basis of her experience and knowledge that I am able to clear all the theoretical and technical hurdles during the development phases of this review paper.

I want to express gratitude to other faculty members of Department of Computer science and Technology for their intellectual support

\section{REFERENCES}

[1] Kaur, Manjeet, and Shailender Gupta. "Comparison of Noise Removal Techniques Using Bilateral Filter." International Journal of Signal Processing, Image Processing and Pattern Recognition 9, no. 2 (2016): 433444.

[2] Aggarwal, Pulkit, Harpreet Kaur, and Navdeep Goel. "Comparative Analysis of Different Algorithm for Removal of High Density Salt and Pepper Noise." (2016). Bandyopadhyay, Aritra, Shubhendu Banerjee, Atanu Das, and Rajib Bag. "A Relook and Renovation over State-of-Art Salt and Pepper Noise Removal Techniques." International Journal of Image, Graphics and Signal Processing 7, no. 9 (2015): 61.

[3] Irum, Isma, Muhammad Sharif, Mussarat Yasmin, Mudassar Raza, and Faisal Azam. "A Noise Adaptive 
Approach to Impulse Noise Detection and Reduction." Nepal Journal of Science and Technology 15, no. 1 (2015): 67-76.

[4] Usharani, L. S., P. Thiruvalar Selvan, and G. Jagajothi. "An Improved De-noising Algorithm for Highly Corrupted Color Videos Using FPGA Based Impulse Noise Detection and Correction Techniques." Indian Journal of Science and Technology 8, no. 7 (2015): 664.

[5] Shujin Zhua, Yuehua Li a, Yuanjiang Li"A PMMW image denoising based on adaptive manifolds and highdimensional mean median filter" elsevier.de/ijleo, September 2015.

[6] Karthickmanoj, R., S. Sinthuja, and N. Manoharan. "Removal of Impulse Noise Using Adaptive Weighted Median Filter." Indian Journal of Science and Technology 7, no. S6 (2014): 61-63.

[7] Lien, Chih-Yuan, Chien-Chuan Huang, Pei-Yin Chen, and Yi-Fan Lin. "An efficient denoising architecture for removal of impulse noise in images." IEEE Transactions on computers 62, no. 4 (2013): 631-643.
[8] Ching-Ta $\mathrm{Lu}$ "Noise reduction using three-step gain factor and iterative-directional median" (2013).

[9] Shi-Jinn Horng a,b, Ling-Yuan Hsu b,c, Tianrui Li a, Shaojie Qiao a, Xun Gong a,Hsien-Hsin Chou d, Muhammad Khurram Khan "Using Sorted Switching Median Filter to remove high-density impulse noises' 'Accepted 25 May 2013

[10] Madhu S. Nair, P.M. Ameera Mol "Direction based adaptive weighted switching median filter for removing high density impulse noise" (2012).

[11] Gang Xiong a, b, n, Tian-HuaiDing c,1 “ADWA: A filtering paradigm for signal's noise removal and feature preservation" Accepted 27 November (2012).

[12] Ravada, Sridevi, Vani Prasanna Kanakala, and Ramya Koilada. "Distinguishing the Noise and image structures for detecting the correction term and filtering the noise by using fuzzy rules." International Journal on Computer Science and Engineering 3, no. 7 (2011): 2754-2764. 